\title{
Wood Materials Defects Detection Using Image Block Percentile Color Histogram and Eigenvector Texture Feature
}

\author{
Weiwei Song ${ }^{1,2}$,Tianyi Chen ${ }^{1,2}$, Zhenghua Gu ${ }^{1,2}$, Wen Gai $^{1,2}$, Weikai Huang ${ }^{1,2}$, \\ Bin Wang ${ }^{1,2, a, *}$ \\ ${ }^{1}$ Facility Design and Instrumentation Institute, China Aerodynamics Research and Development
Center, Mianyang 621000, China
}

${ }^{2}$ State Key Laboratory of Aerodynamics, China Aerodynamics Research and Development Center, Mianyang 621000, China

anudtwangbing@163.com

\begin{abstract}
Keywords: Wood materials, defects detection, percentile color histogram, singular value
\end{abstract} decomposition, image block feature, support vector machine (SVM).

\begin{abstract}
To automatic detect wood surface defects, a method based on image block percentile color histogram and eigenvector texture feature classification is proposed. Firstly, a wood surface image is divided into several same size image blocks. Secondly, for each image block, a percentile color histogram is calculated as image block color feature. Meanwhile, singular value decomposition (SVD) is adopted to extract k-max eigenvectors as image block texture feature. Then the percentile color histogram and eigenvector texture feature is combined to a feature vector for image block representation. Finally, a support vector machine (SVM) classifier is trained and used to determine which image block is sound or defect wood. The experimental results show that the proposed method can effectively detect wood surface defects, especially the knot type defects.
\end{abstract}

\section{Introduction}

Nowadays, wood is recognized green material, compared with the world widely used materials such as steel, cement, and plastic. However, the supply of wood far cannot satisfy the market demand. A reasonable wood use is imperative.

As shown in Fig.1, wood defects will obviously damage wood quality, and reduce the value of wood products. It is very important to research wood defects automatic detection technology. In the last century, most researchers pay more attention on wood defects detection using ultrasonic, X-ray and microwave. However, X-ray method needs the expensive X-ray camera equipment, and its radiation is dangerous for workers. While the performance of microwave method is affected by the moisture content in wood.

In recent years, the wood surface defects detection method based on visual information has been widely concerned by scholars, and emerged a large number of algorithms. Since the color and texture from would and defect wood images is obviously difference. Using wood visual information for wood defects detection is feasible. To detect wood defects, most researchers ${ }^{[1-4]}$ use image segmentation algorithms with color or texture information. In image segmentation result, the wood pixels are divided into two regions: would and defect wood. However, image segmentation is one of the most difficult task in image processing area. Although many image segmentation methods have been proposed in the last decade. Due to the wood color and texture variety, it is very difficult to discriminate would and defect wood with image segmentation.

In this paper, we use an image block classification framework for wood surface defects detection. It uses image block feature replace image pixel for defects detection. Firstly, we divide wood image into several same size image blocks. Second, we extract color and texture features from each image block. Finally, a support vector machine (SVM) classifier is trained and used for image block classification. In the labeled results, the world and defect wood are separated. The contribution of this paper is that we provide a novel method to extract image block feature. Considering that the world and defect woods are different in color and texture, a combine feature vector with percentile color 
histogram and eigenvector texture feature is used for image block representation. The experimental results show that the proposed method is effective for wood defects detection.

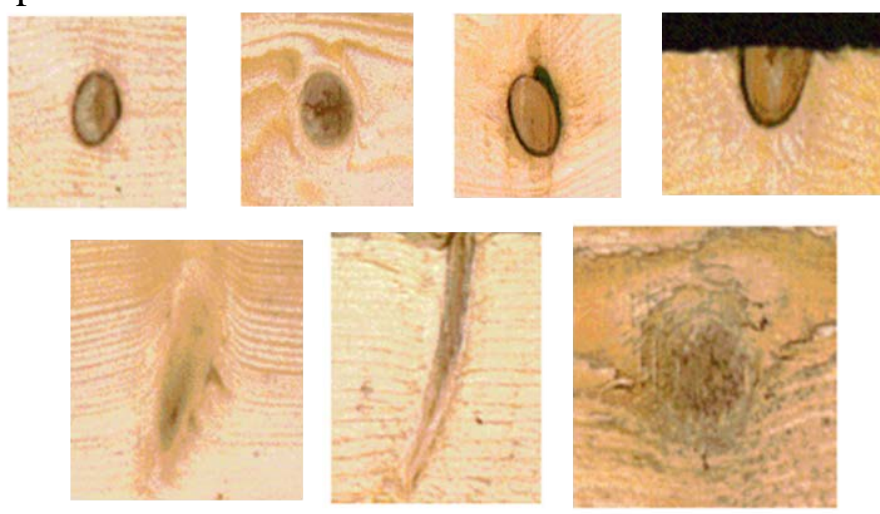

Fig.1. Examples of wood defect samples

\section{Our method}

Wood defects detection based on image block classification. The image block classification method is applied in following way. A wood image $\boldsymbol{I}$ is divided into rectangular regions $\left(a_{n}\right), n=1, \ldots, N$ of size by $s \times s$ pixels. A set of features is calculated from these regions, and they are classified to categories relevant to would or defect wood. An example of image block classification method is shown in Fig.2. In Fig.2 a), the wood image has been divided into rectangular regions of 32 x 32 pixels. A set of color and texture features is calculated for each rectangular region. Then the image blocks are classified to defect or would wood. The resulting regions containing possible defects are shown in Fig. 2 b).
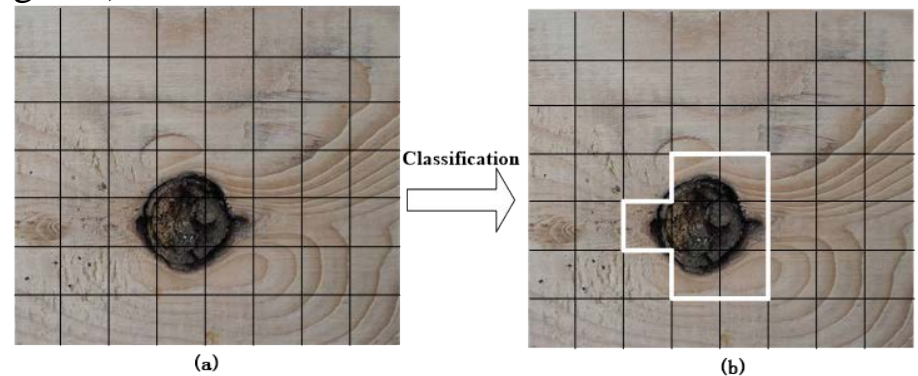

(b)

Fig.2 The principle of image block classification method.

Image block features. Percentile color histogram. In Matti Niskanen's works ${ }^{[5]}$, the percentile color histogram is calculated from a cumulative histogram $C_{k}(x)$, which is defined as a sum of the normalized histogram $\boldsymbol{P}_{\boldsymbol{k}}(\boldsymbol{x})$ of color channel $\boldsymbol{k}$ of all the values that are smaller than $\boldsymbol{x}$ or equal to $\boldsymbol{x}$. Finding a value for a percentile feature is finding the $x$, when $C_{k}(x)$ is known, requiring an inverse function of $C_{k}(x)$. Let us denote the percentile feature value with $F_{k}(y)$, we get the relation

$$
F_{k}(y)=C_{k}^{-1}(y)=x
$$

Where $y$ is a value of the cumulative histogram in the range [0\%, 100\%].

An example of color histogram, cumulative histograms and percentile color histogram in $\mathrm{R}$ channel are shown in Fig.3. Where, take the determination of the 10th percentile $F_{R}(\mathbf{1 0} \%$ ) (called later $10 \%$ percentile) of the red channel. Now $y=10 \%$ and from the figure we can read (drawn by a thick line) the $10 \%$ percentile feature value $F_{R}(\mathbf{1 0 \%})=\mathbf{1 2 0}$. At this point, $10 \%$ of the histogram distribution is to the left of it.

Here, we use the $0.2 \%, 1 \%, 10 \%, 50 \%, 95 \%$ for 3 color channels. And they form a feature vector

$\bar{f}=\left[f_{1}, \ldots, f_{15}\right]$, where the elements $f_{i}$ in this case are the percentile features $F_{k}(y)$ for any desired color channel $k$ and cumulative values $y$. Generally, a feature vector may contain also features other than color percentile features. 
To use pure centiles, proper color calibration is needed. Invariant features against the shift of the histogram can be found by calculating differences of two percentiles $f=\left[F_{k}\left(y_{1}\right)-F_{k}\left(y_{2}\right)\right]$. Invariance against both the shift and the width of the histogram can be achieved by normalizing a difference percentile feature by the difference of the maximum and minimum percentile values of the histogram
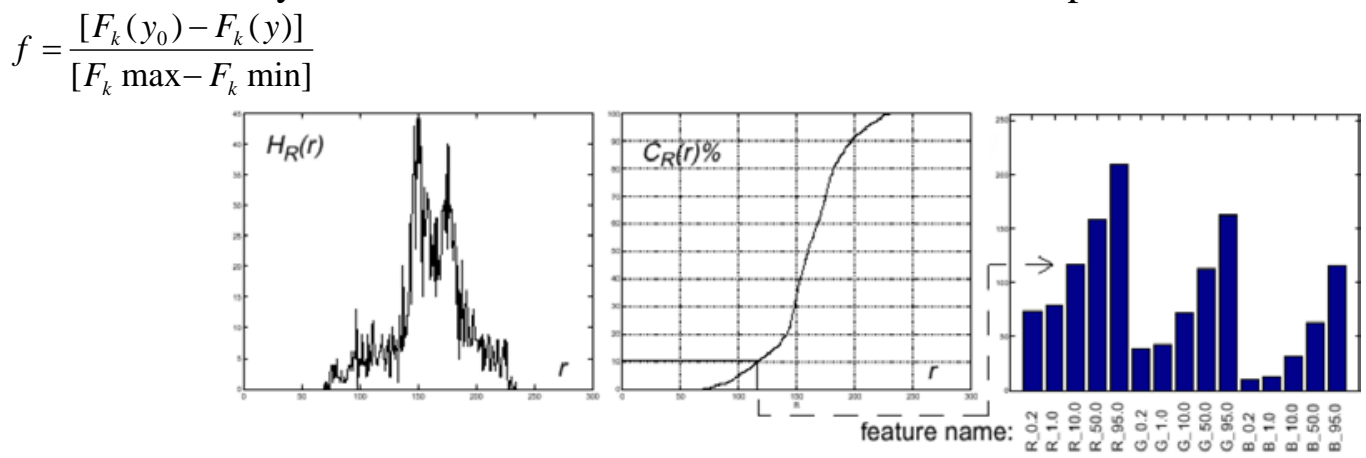

Fig.3. An example of percentile color histogram in R channel [5].

In practice, the maximum and minimum percentiles may appear unreliable for this purpose, since saturated or noise pixels may cause them to lie at the maximum and minimum possible histogram bin value. Instead, it is safer to use $95 \%$ and $5 \%$ percentiles as the maximum and minimum values, for example.

It is obvious that the percentile color histogram features are sensitive to intensity changes in RGB color space. In order to handle with this problem, the color space selected was HSV for the reason that, considering the histograms it was possible to extract enough features that gave the system the capacity to distinguish images that looked similar to human eye ${ }^{[6,7]}$. Also, these color spaces are more uniform than RGB and are less sensitive to illumination drifts. Color space HSV is a device dependent model ${ }^{[7,8]}$ that makes use of three concepts: Hue; Saturation and Value, and is a nonlinear transformation of RGB color space (Fig.4). Hue corresponds to the dominate wavelength of the scene spectrum and ranges from $0^{\circ}$ to $360^{\circ}$. Saturation is related with the purity of the color, meaning the quantity of white. It ranges from 0 (no color) to 100 (pure color). Value is related with the brightness of the color, meaning the intensity of the electromagnetic energy, and it ranges from 0 (black) to 100 (white).

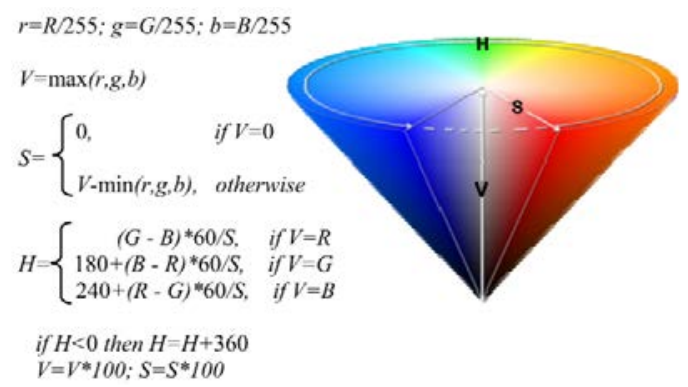

Fig. 4. Conical representation of the HSV model and RGB to HSV conversion equations.

Eigenvector texture feature. For an image block matrix, eigenvector is very useful for texture representation. For example, in face recognition, to extract the illumination invariant features and reduce the dimension of face images. Eigenvector is extract and used for face representation. Inspired from the Eigneface methods ${ }^{[9]}$, we adopted eigenvector for wood image block texture representation.

Usually, texture feature is extracted on gray scale image. Here, we firstly convert wood image color from RGB to Gray:

$\mathrm{G}=0.299 \mathrm{R}+0.587 \mathrm{G}+0.114 \mathrm{~B}$

As shown in Fig.5, for each image block $\left(a_{n}\right)$ with the size of $s \times s$ pixels, extract s columns and $s$ rows to make two matrix $M_{c}$ and $M_{r}$. Then apply the singular value decomposition (SVD) to calculate the eigenvectors $E_{c}$ (and $E_{r}$ ) from $M_{c} M_{c}{ }^{T}$ (and $M_{r} M_{r}{ }^{T}$ ) respectively. After that, each eigenvectors in $E_{c}$ are ordered descend upon eigenvalue. A same operation is also carried out on $E_{r}$. Finally, we select the 1st an and 2nd max eigenvector in $E_{c}$ (and $E_{r}$ ) to combine the image block Eigenvector texture feature: $\hat{f}=\left[E_{c}^{1}, E_{c}^{2}, E_{r}^{1}, E_{c}^{2}\right]$. 


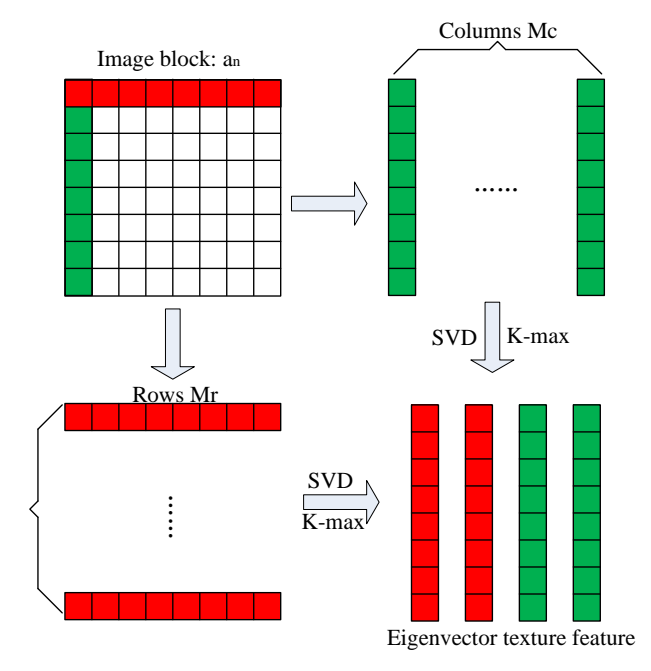

Fig. 5 the illustration for image block eigenvector texture feature extraction

In fact, the vector texture feature extraction is represent the image block in a lower dimensional space. According to PCA analysis, the vector texture feature in this paper provides an optimal in the mean square error sense representation of $M_{c}$ and $M_{r}$.

Features combine. The image block is representation with the combine feature of color percentile histogram $\bar{f}$ and texture vector feature $\hat{f}: f=[\bar{f}, \hat{f}]$. Since the value range of percentile color histogram and eigenvector feature is different. Before feature combine, a normalization is implemented both for $\bar{f}$ and $\hat{f}$. Here $f$ is $(15+4 s)$ dimensions feature vector. Obvious the dimension is lower than the original image blocks. For example, a $32 * 32$ pixels image block in RGB color space, its dimension is 3072. While the dimension is 143 in $f$.

Table 1. The number of manually labeled defects in the sample sets.

\begin{tabular}{|c|c|c|}
\hline & Number of samples in Set1 & Number of samples in Set2 \\
\hline Sound knot & 911 & 268 \\
\hline Dry knot & 1343 & 528 \\
\hline Black knot & 63 & 31 \\
\hline crack & 355 & 86 \\
\hline Resin pocket & 70 & 40 \\
\hline Other defects & 92 & 33 \\
\hline Total & 2834 & 986 \\
\hline \multicolumn{3}{|c|}{ Table 2. Error escapes of defect. } \\
\hline & Number of samples & Error escape rate \\
\hline Sound knot & 268 & 0.10 \\
\hline Dry knot & 528 & 0.07 \\
\hline Black knot & 31 & 0.12 \\
\hline crack & 86 & 0.55 \\
\hline Resin pocket & 40 & 0.15 \\
\hline Other defects & 33 & 0.28 \\
\hline
\end{tabular}

\section{Experiments and results}

A set of 120 images of pine wood board surface sides was used as the sample material from VTT Building Technology Wood dataset. The defects on the images were manually selected and labeled. The images were divided into two sample sets, the first one containing 80 images (set1) and the second one 40 images (set2). Set1 was used for training and set2 for test purposes. The number of manually selected and labeled defect samples in the sample sets is shown in Table 1. The category other defects consists of 14 different defect types represented by a very small number of samples and were therefore grouped together.

The LIBSVM ${ }^{[11]}$ software is adopted is adopted for image block classification. And the nonlinear Gaussian kernel is used. In this experiments, the image block size is set as $64 * 64$. After finished SVM 
classifier training. The classifier is used to predict the wood image block as would or defect. One of the classification results are shown in Fig.6. The error escapes of defects are shown in Table 2. It shows that the detection effect is very ideal.

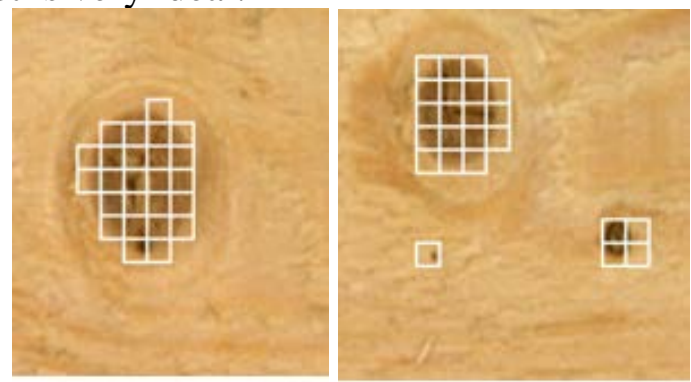

Fig.6 one example of wood defect detection result of the proposed method

\section{Conclusion}

In this paper, a wood defects detection method based on image block percentile color histogram and eigenvector texture feature classification is proposed. Different from the existing image segmenting methods. It divides wood images into blocks and classifies each block as would or defect wood upon the image block percentile color histogram and eigenvector texture features. The experimental results show that the proposed method can effectively detect wood surface defects, especially the knot type defects.

While, the eigenvector texture feature is not rotation invariant. In the future work, we will improved the eigenvector texture feature extraction method with ring sampling.

\section{References}

[1] Funck J W, Zhong Y, Butler D A, et al. Image segmentation algorithms applied to wood defect detection[J]. Computers and electronics in agriculture, 2003, 41(1) p.157-179.

[2] Ruz G A, Estévez P A, Perez C A. A neurofuzzy color image segmentation method for wood surface defect detection [J]. Forest products journal, 2005, 55(4) p.52-58.

[3] Ruza’b G A, Estéveza P A. Image segmentation using fuzzy min-max neural networks for wood defect detection[C]//Intelligent Production Machines and Systems-First IPROMS Virtual Conference: Proceedings and CD-ROM set. Elsevier, 2005 .p.183.

[4] Kumar A, Pang G K H. Defect detection in textured materials using Gabor filters [J]. Industry Applications, IEEE Transactions on, 2002, 38(2) p.425-440.

[5] Silvén O, Niskanen M, Kauppinen H. Wood inspection with non-supervised clustering [J]. Machine Vision and Applications, 2003, 13(5-6) p. 275-285.

[6] Benjamin D. Zarit, Boaz J. Super, Francis K. H. Quek, Comparison of Five Color Models in Skin Pixel Classificatio,in Proceedings of the International Workshop on Recognition, Analysis, and Tracking of Faces and Gestures in Real-Time Systems, p.58, September 1999.

[7] Raphael Gonzalez, Richard E. Woods, Digital Image Processing, 2nd ed. Prentice Hall Press, p. 295. 2002, ISBN 0-201-18075-8.

[8] Donald Hearn, M. Pauline Baker, Computer Graphics Prentice Hall International, pp. 302-205, 1986, ISBN 0-13-165598-1.

[9]Turk M, Pentland A. Eigenfaces for recognition [J]. Journal of cognitive neuroscience, 1991, 3(1) p. 71-86.

[10] LIBSVM: http://www.csie.ntu.edu.tw/ cjlin/libsvm/index.html 